\title{
Predictive value of phosphorylated mammalian target of rapamycin for disease-free survival in breast cancer patients receiving neoadjuvant chemotherapy
}

\author{
SHUO WANG ${ }^{1}$, YIQUN SUN ${ }^{2}$, ANNING HE $^{3}$, CAIWEI ZHENG ${ }^{4}$ and XINYU ZHENG ${ }^{1,3}$ \\ ${ }^{1}$ Department of Breast Surgery, First Affiliated Hospital, China Medical University, Shenyang, Liaoning 110001; \\ ${ }^{2}$ Department of Burns, General Hospital of Benxi Iron and Steel Company, Benxi, Liaoning 117000; \\ ${ }^{3}$ Lab 1, Cancer Institute, China Medical University, Shenyang, Liaoning 110001, P.R. China; \\ ${ }^{4}$ Department of Biology, Brandeis University, Waltham, MA 02453, USA
}

Received December 21, 2013; Accepted July 25, 2014

DOI: $10.3892 / \mathrm{ol} .2014 .2551$

\begin{abstract}
The mammalian target of rapamycin(mTOR)/eukaryotic translation initiation factor 4E-binding protein 1 (4E-BP1) pathway plays a critical role in cell growth, survival and angiogenesis, and has been demonstrated to correlate with human epidermal growth factor receptor 2 (HER2) status. Neoadjuvant chemotherapy (NAC), also known as preoperative therapy, is now well established in the treatment of inoperable locally advanced and inflammatory breast cancer. In vitro study has shown that mTOR inhibitors, together with cytotoxic agents, exhibit tumor cell killing activity. A number of non-randomized studies in HER2-positive trastuzumab-resistant metastatic breast cancer have revealed the antitumor activity of mTOR inhibitors when used together with standard chemotherapy plus trastuzumab. In the present study, the expression levels of phosphorylated (p)-mTOR and p-4E-BP1 were analyzed in breast cancer patients prior to and following NAC, to determine whether p-mTOR and p-4E-BP1 affect the response to NAC and the subsequent survival. Formalin-fixed, paraffin-embedded tissues representing matched pairs of core biopsy (pre-NAC) and surgical specimen (post-NAC) from 83 patients with invasive ductal carcinomas were collected. Immunohistochemistry was performed to evaluate the expression of p-mTOR and p-4E-BP1 using a semi-quantitative scoring system by two pathologists. It was found that the expression of p-mTOR and p-4E-BP1 was downregulated following NAC. The decrease in mTOR expression following NAC was found to positively correlate with HER2 expression
\end{abstract}

Correspondence to: Professor Xinyu Zheng, Department of Breast Surgery, First Affiliated Hospital, China Medical University, 155 North Nanjing Street, Shenyang, Liaoning 110001, P.R. China E-mail: xyzheng@mail.cmu.edu.cn

Key words: phosphorylated-mammalian target of rapamycin, phosphorylated-eukaryotic translation initiation factor 4E-binding protein, neoadjuvant chemotherapy, predictive value and the reduction of tumor sizes. The high expression of p-mTOR and p-4E-BP1 in pre-NAC specimens was associated with poor disease-free survival (DFS). Furthermore, the high expression of p-mTOR in post-NAC specimens was associated with poor DFS, regardless of whether the expression was high or low in the pre-NAC specimens. In conclusion, NAC was found to decrease the expression levels of p-mTOR and $\mathrm{p}-4 \mathrm{E}-\mathrm{BP} 1$. The $\mathrm{p}-\mathrm{mTOR}$ expression post-NAC may potentially serve as a predictor for DFS. However, further study is required to clarify the mechanism and to evaluate the predictive value of the phosphatidylinositol 3-kinase/Akt/mTOR/4E-BP1 pathway in NAC.

\section{Introduction}

The mammalian target of rapamycin (mTOR), a highly conserved serine/threonine protein kinase, is critical for cell growth, survival and angiogenesis (1). Members of the epidermal growth factor receptor family, including human epidermal growth factor receptor 2 (HER2), use the phosphatidylinositol 3-kinase (PI3K)/Akt/mTOR pathway to promote cell growth and survival (2). mTOR is predominantly controlled by the PI3K/Akt pathway and can be activated by Akt-mediated phosphorylation $(3,4)$. mTOR has two main downstream messengers, the 40S ribosomal S6 kinase (S6K1) and the eukaryotic translation initiation factor 4E-binding protein 1 (4E-BP1) (5), both of which are activated via phosphorylation by phosphorylated (p)-mTOR.

The eukaryotic initiation factor $4 \mathrm{E}$ (eIF4E) complex is an initiation factor on the 5' cap structure of mRNA that recruits the small ribosomal subunit to mRNA. It contains three initiation factors, eIF4E, eIF4G and eIF4A $(2,6)$. To assemble the eIF4E complex, eIF4E first binds to the 5' cap to recruit eIF4G and eIF4A. However, 4E-BP1 inhibits eIF4G binding to eIF4E. The p-4E-BP1 loses its ability to bind to eIF4E and allows the eIF4E complex to bind to the cap structure of mRNA (7-9), subsequently initiating the protein translation. It has been shown that eIF4E expression is associated with patient survival following anthracycline chemotherapy treatment, and the influence of eIF4E on cancer survival is modulated 
substantially by 4E-BP1 $(10,11)$. The PI3K/Akt/mTOR/4E-BP1 pathway has also been found to correlate with HER2, and it may be used as a predictive marker for patient prognosis (2). Another downstream factor, S6K1, is associated with the translational machinery and has been demonstrated to predict poor prognosis in hormonal receptor-positive breast cancer patients $(12,13)$.

Neoadjuvant chemotherapy (NAC), also known as preoperative therapy, uses chemotherapy as the initial treatment of malignant tumors, followed by surgery or other therapies. NAC is now well established in the treatment of inoperable locally advanced and inflammatory breast cancer. It is also used in operable breast cancer treatment in order to obtain clinical and pathological response to NAC, or to downstage tumors to allow breast-conserving surgery $(14,15)$. A large number of studies have demonstrated the efficacy of NAC in primary operable and locally advanced breast cancer patients, as well as patients who have achieved pathological complete response (pCR), which is regarded as a good surrogate predictor of disease-free survival (DFS) and overall survival (OS) (16-19). However, few molecular markers are available to predict the NAC responses or survival gains. It has been demonstrated that PI3K/Akt/mTOR is commonly deregulated in human cancers $(20,21)$, due to the mutation of PIK3CA, Akt and phosphatase and tensin homolog (PTEN), or the loss of PTEN (22-25). It has also been demonstrated that the combination of mTOR inhibitors with cytotoxic agents can exert synergistic antiproliferative activity in in vitro studies, irrespective of HER2 status (26). A number of non-randomized studies in HER2-positive trastuzumab-resistant metastatic breast cancer have shown the antitumor activity of mTOR inhibitors when used together with standard chemotherapy plus trastuzumab $(27,28)$. However, a study evaluating the addition of mTOR inhibitors to paclitaxel treatment in HER2-negative patients suggested that supplementing paclitaxel treatment with everolimus did not significantly improve pCR rates compared with those of paclitaxel alone (29). A number of other clinical trials have been initiated to identify the most beneficial therapeutic strategies to include mTOR inhibitors for different patient subgroups (30).

\section{Materials and methods}

Patients and tissues. In total, 83 primary breast cancer patients treated with NAC at the First Hospital of China Medical University (Shenyang, China) between 2007 and 2010 were selected. Preoperative chemotherapy was performed as follows: 37 patients were administered docetaxel $\left(75 \mathrm{mg} / \mathrm{m}^{2}\right)$ with platinum (TP; $100 \mathrm{mg} / \mathrm{m}^{2}$ ) or cyclophosphamide (TC; $1.0 \mathrm{~g}$ ) every three weeks for three to five cycles, while the other 46 patients received 5-fluorouracil (1.0 g), epirubicin $\left(80 \mathrm{mg} / \mathrm{m}^{2}\right)$ and cyclophosphamide (CEF; $\left.1.0 \mathrm{~g}\right)$ every three weeks for three to four cycles. Patients were followed-up for a median of 45 months after their initial cancer surgery. Relevant clinical and pathological parameters are described in Table I. Archival formalin-fixed, paraffin-embedded breast tissues were collected from core biopsies (pre-NAC) and matched resection tissues (post-NAC). Six patients achieved pCR. All of the carcinomas had been histologically confirmed as invasive breast cancer according to the criteria of the World Health Organization (31) and the molecular subtypes of breast carcinoma were identified.

Immunohistochemical staining. Immunohistochemical examination was performed on $4-\mu \mathrm{m}$ formalin-fixed, paraffin-embedded sections. Briefly, following deparaffinization and rehydration, the endogenous peroxidase activity was blocked using $3 \% \mathrm{H}_{2} \mathrm{O}_{2}$ (reagent A; UltraSensitive ${ }^{\mathrm{TM}} \mathrm{SP}$ IHC kit; Maxim Biotech Inc., Fuzhou, China). Next, antigen retrieval was performed and normal serum was applied to the sections to block non-specific antibody binding (reagent B; UltraSensitive $^{\mathrm{TM}}$ SP IHC kit; Maxim Biotech Inc.). Sections were then incubated overnight at $4^{\circ} \mathrm{C}$ with the primary antibodies. A goat anti-rabbit polyclonal antibody against mTOR (phospho S2448) was used for p-mTOR at a dilution of 1:500 (ab131538; Abcam, Cambridge, UK), and a goat anti-rabbit polyclonal antibody against eIF4EBP1 (phospho Thr36) was used for p-4EBP1 at a dilution of 1:200 (ab47365; Abcam). Following overnight incubation at $4^{\circ} \mathrm{C}$, sections were incubated for $15 \mathrm{~min}$ with the secondary antibody solution (reagent C; UltraSensitive ${ }^{\mathrm{TM}}$ SP IHC kit; Maxim Biotech Inc.). The sections were then incubated with streptavidin-perosidase (reagent D; UltraSensitive ${ }^{\mathrm{TM}}$ SP IHC kit; Maxim Biotech Inc.) for $15 \mathrm{~min}$ and stained with 3,3-diaminobenzidine. Finally, sections were counterstained with hematoxylin for $5 \mathrm{~min}$ and mounted. Negative controls were processed with normal rabbit serum (Dako, Carpinteria, CA, USA) in place of the primary antibody. Positive controls were performed using breast cancer tissue sections that had shown strong staining for the respective protein during antibody optimization. This study was approved by the ethics committee of the First Affiliated Hospital (Shenyang, China) and written informed consemt was obtained from all patients.

Evaluation of immunohistochemistry. The immunohistochemical staining results were evaluated and scored independently in a blinded manner by two pathologists. Cases of disagreement were reviewed jointly to obtain a consensus score. The score was the average of 10 distinct high-power fields observed under the 40x objective. The staining was considered positive when cytoplasmic and/or membranous staining was observed in the malignant cells, and the staining was evaluated using a semi-quantitative scoring system considering the extent and intensity. The percentage of cells stained were scored as follows: 0 , no cells stained; $1,1-10 \%$ of cells stained; $2,11-50 \%$ of cells stained; $3,51-80 \%$ of cells stained; and $4,>80 \%$ of cells stained. Staining intensity was scored as follows: 0, negative; 1 , weak; 2 , moderate; and 3, strong. The two parameters were multiplied, resulting in an individual immunoreactivity score ranging between 0 and 12 for every case. The six patients who achieved pCR were regarded as negative post-NAC.

Statistical analysis. Statistical analyses were performed using SPSS v.19.0 (SPSS, Inc., Chicago, IL, USA). Wilcoxon signed-rank test was used to evaluate the independence between two groups of matched samples, and Mann-Whitney U test was used to assess the independence between two independent samples without any distribution assumption. Spearman's correlation coefficients were used to reveal a correlation between two continuous variables. Receiver Operating 
Table I. Clinical and pathological features of the patients $(n=83)$.

\begin{tabular}{|c|c|}
\hline Characteristic & $\mathrm{n}(\%)$ \\
\hline \multicolumn{2}{|l|}{ Age, years } \\
\hline$\leq 45$ & $49(59.0)$ \\
\hline$>45$ & $34(41.0)$ \\
\hline \multicolumn{2}{|c|}{ Pre-NAC stage (based on ultrasound) } \\
\hline $\mathrm{T} 2$ & $41(49.4)$ \\
\hline $\mathrm{T} 3$ & $24(28.9)$ \\
\hline $\mathrm{T} 4$ & $18(21.7)$ \\
\hline \multicolumn{2}{|c|}{ Post-NAC stage (based on resection pathology) } \\
\hline T0 & $6(7.2)$ \\
\hline $\mathrm{T} 1$ & $23(27.7)$ \\
\hline $\mathrm{T} 2$ & $33(39.8)$ \\
\hline $\mathrm{T} 3$ & $12(14.5)$ \\
\hline $\mathrm{T} 4$ & $9(10.8)$ \\
\hline \multicolumn{2}{|l|}{ Tumor size change ${ }^{a}$} \\
\hline Increase & $8(9.6)$ \\
\hline Decrease & $69(83.1)$ \\
\hline $\mathrm{pCR}$ & $6(7.2)$ \\
\hline \multicolumn{2}{|l|}{ NAC regimen } \\
\hline $\mathrm{TP}$ & $32(38.6)$ \\
\hline $\mathrm{TC}$ & $15(18.1)$ \\
\hline $\mathrm{CEF}$ & $36(43.4)$ \\
\hline Positive axillary metastasis & $61(73.5)$ \\
\hline Estrogen receptor-positive & $37(44.6)$ \\
\hline Her2-positive & $51(61.4)$ \\
\hline \multicolumn{2}{|l|}{ Surgery } \\
\hline Breast conserving & $2(2.4)$ \\
\hline Mastectomy & $81(97.6)$ \\
\hline Follow-up, months ${ }^{\mathrm{b}}$ & $45(32-78$ \\
\hline Recurrence & $28(33.7)$ \\
\hline Mortality & $23(27.7)$ \\
\hline
\end{tabular}

${ }^{a}$ Change between the initial size at ultrasound and final size at resection pathology; ${ }^{b}$ Median (range). NAC, neoadjuvant chemotherapy; pCR, pathological complete response; TP, docetaxel + platinum; TC, platinum + cyclophosphamide; CEF, 5-fluorouracil + epirubicin + cyclophosphamide.

Characteristic (ROC) curve analyses were used to select cut-off values (giving the highest combined sensitivity and specificity) to dichotomize pre- and post-NAC expression scores for the endpoint of DFS. DFS was recorded from the date of surgery to the date of relapse or last follow-up, and estimated using the Kaplan-Meier analyses. The statistical significance of the differential survival was assessed using the log-rank (score) test. Additionally, multivariate Cox regression analysis was performed taking into account the pre-NAC expression of p-mTOR and p-4E-BP1, and post-NAC expression of p-mTOR, as well as other clinicopathological features, including tumor grade, receptor status, pre-NAC tumor stage and axillary metastasis. All P-values presented are two-sided, and $\mathrm{P} \leq 0.05$ was considered to indicate a statistically significant difference.

\section{Results}

p-mTOR and p-4E-BPI are downregulated following NAC and correlate with each other in pre-NAC samples. The expression of p-mTOR and p-4E-BP1 were examined in the tumors of 83 cases of breast cancer patients. The patients who had achieved pCR were not examined post-NAC. Tumors were obtained from diagnostic biopsies pre-NAC and surgical resections post-NAC. Table I shows the clinical and pathological features of the patient cohort. The staining of p-mTOR was predominantly cytoplasmic, and present in 71/83 cases $(85.5 \%)$ and $58 / 83$ cases $(69.9 \%)$ pre-NAC (Fig. 1A) and post-NAC (Fig. 1B), respectively. The p-4E-BP1 was detected in the nucleus and cytoplasm, with positive rates of $\mathrm{p}-4 \mathrm{E}-\mathrm{BP} 1$ in $69 / 83$ cases $(83.1 \%$ ) and $58 / 83$ cases $(69.9 \%)$ pre-NAC (Fig. 1C) and post-NAC (Fig. 1D), respectively. The scores and their distributions are shown in Fig. 2. Scores were lower for p-mTOR (51/83 cases; 61.4\%) and p-4E-BP1 (56/83 cases; $67.5 \%$ ) in post-NAC samples than in the matched pre-NAC samples, indicating a decrease in their expression in response to NAC. Wilcoxon signed-rank test showed significant differences between pre- and post-NAC scores $(\mathrm{P}<0.001$ for $\mathrm{p}-\mathrm{mTOR}$ and $\mathrm{p}-4 \mathrm{E}-\mathrm{BP} 1)$. It was also examined whether a correlation exists between the expression of p-mTOR and p-4E-BP1 preand post-NAC, as well as for the expression change following treatment (pre-NAC minus post-NAC level). The expression of p-mTOR and p-4E-BP1 were found to significantly correlate with each other in pre-NAC samples (Spearman's Q analyses, $\mathrm{P}=0.004)$, which is consistent with previous studies $(2,32,33)$. However, the two factors were not found to correlate with each other in post-NAC samples, indicating that chemotherapy may have changed the expression of the two factors to some degree. No significant correlations were found between the changes of p-mTOR and p-4E-BP1 following NAC. As would be expected, the pre- and post-NAC levels were significantly associated with the expression change of the respective factor.

Decrease of p-mTOR expression following NAC positively correlates with HER2 expression and diminishing tumor size. Pre-NAC expression, post-NAC expression and the expression change following NAC of p-mTOR and p-4E-BP1 was evaluated to identify correlations with patient or tumor characteristics, including patient age at diagnosis, tumor grade and stage, estrogen receptor status, and HER2 status of tumors from core biopsy at diagnosis, as well as the tumor stage and presence of axillary metastases from resection pathology. Spearman's @ analyses were performed, and not only did the expression of p-mTOR and p-4E-BP1 in pre-NAC samples correlate with HER2 [ $\mathrm{Q}$ coefficient, $0.181(\mathrm{P}=0.05)$ for p-mTOR; @ coefficient, $0.193(\mathrm{P}=0.04)$ for $\mathrm{p}-4 \mathrm{E}-\mathrm{BP} 1]$, which is consistent with the previous study, but the change of mTOR expression was also found to significantly correlate with HER2 ( $\mathrm{Q}$ coefficient, 0.275; $\mathrm{P}=0.006$ ). Next, the changes of $\mathrm{p}-\mathrm{mTOR}$ in HER2-positive and -negative groups were compared, and the expression of mTOR was found to decrease significantly following treatment with NAC in the HER2-positive group. The changes of p-mTOR expression had median values of 4 in the HER2-positive group and 1 in HER2-negative group. The Mann-Whitney U test was used to assess the differences between the two groups, and a P-value of 0.041 was 

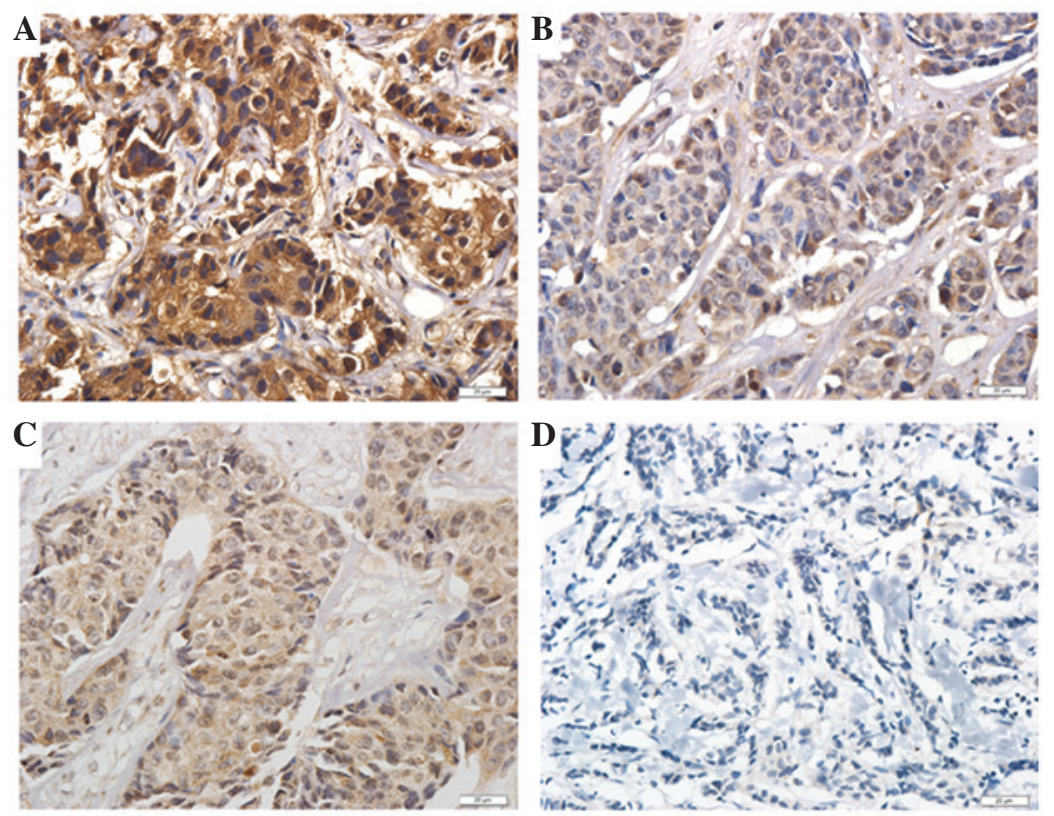

Figure 1. Representative staining images of phosphorylated-mammalian target of rapamycin in matched breast tumor tissues (A) pre- and (B) post-NAC chemotherapy, and phosphorylated-eukaryotic translation initiation factor 4E-binding protein (C) pre- and (D) post-NAC chemotherapy. NAC, neoadjuvant (staining intensity, A, strong; B, moderate; C, moderate; D, negative). Bar, $20 \mu \mathrm{m}$; magnification, $\mathrm{x} 400$.
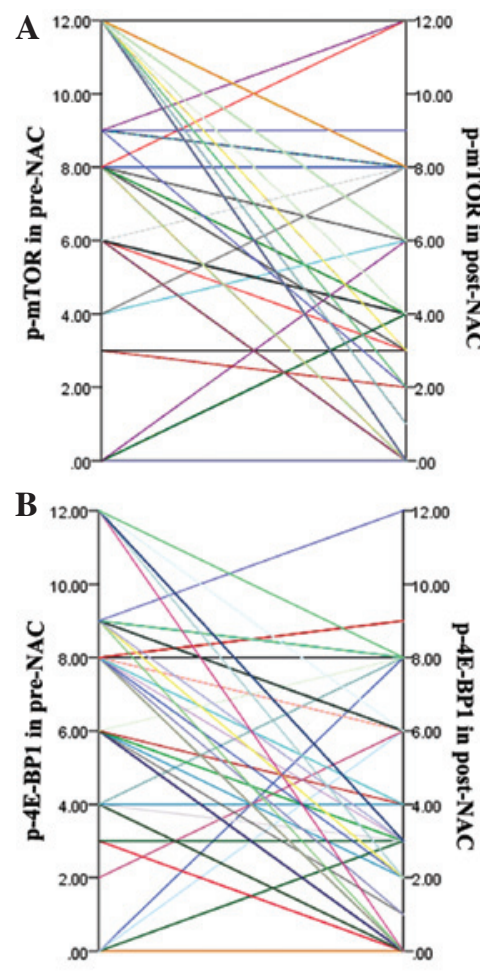
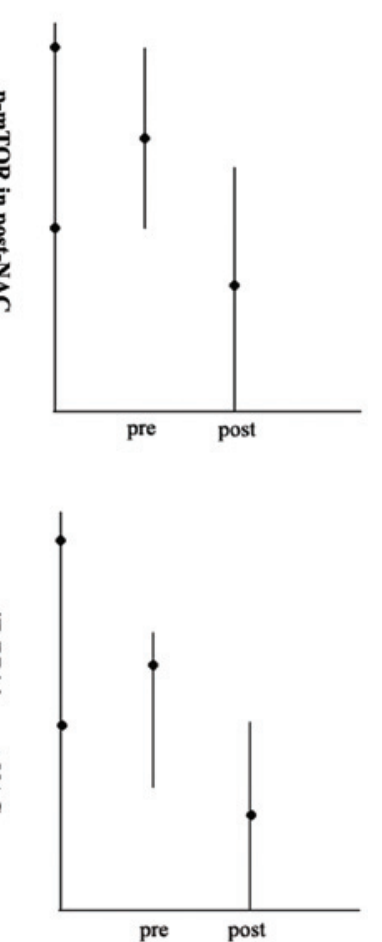

Figure 2. Expression levels of (A) p-mTOR and (B) p-4E-BP1 in matched breast tumor tissues pre- and post-NAC. Left panels show the distribution of the scores of evaluation of the immunohistochemistry pre- and post-NAC. Right panels show the median values (central marker) with interquartile ranges (bars) pre- and post-NAC. The median expression of p-mTOR and p-4E-BP1 were decreased signifcantly following chemotherapy. NAC, neoadjuvant chemotherapy; p-mTOR, phosphorylated-mammalian target of rapamycin; p-4E-BP1, phosphorylated-eukaryotic translation initiation factor 4E-binding protein 1 .

obtained, suggesting some degree of cross-talk between the PI3K/Akt/mTOR-related pathways and HER2. The expression of p-mTOR and p-4E-BP1 pre- and post-NAC was also

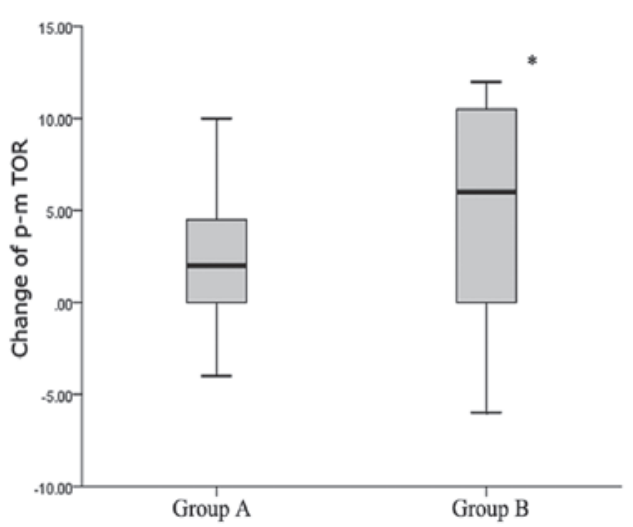

Figure 3. Median values with interquartile ranges of mammalian target of rapamycin changes in groups A and B. Patients whose tumor sizes diminished by $<1 \mathrm{~cm}$, showed no change or increased were defined as group A, while patients whose tumor sizes diminished by $>1 \mathrm{~cm}$ or achieved pathological complete response were defined as group B. The Mann-Whitney U test was used to determine significant differences between groups $\mathrm{A}$ and $\mathrm{B}(\mathrm{P}=0.033)$. The decreased p-mTOR expression was more significant in patients whose tumor size had decreased by $>1 \mathrm{~cm}$ or who had achieved a complete pathological response.p-mTOR, phosphorylated-mammalian target of rapamycin.

evaluated, as well as the expression change of the two factors to identify correlations with tumor size. Therefore, patients were classified into different groups according to tumor size; patients with tumors that had diminished by $<1 \mathrm{~cm}$, showed no change, or had increased were defined as group A, while patients whose tumor sizes had diminished by $>1 \mathrm{~cm}$, or patients who had achieved PCR were defined as group B. The only significant correlation was found between the expression change of mTOR and diminishing tumor size. The median values of expression change of mTOR were 2 in group A and 6 in group B (Fig. 3). The Mann-Whitney U test showed a significant difference between groups $\mathrm{A}$ and $\mathrm{B}(\mathrm{P}=0.033)$. No 

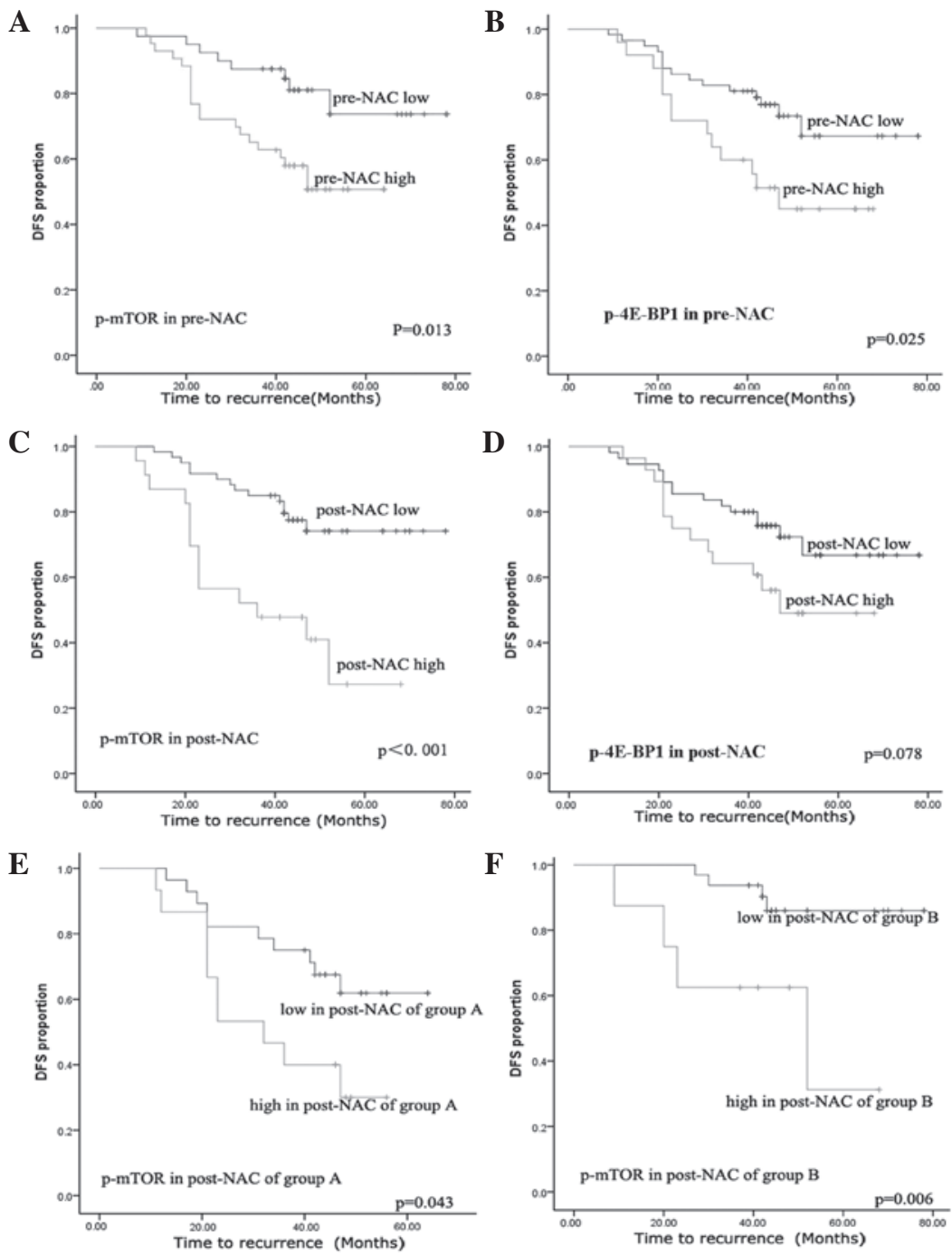

Figure 4. Kaplan-Meier survival analyses of disease-free survival in different patient groups. (A-D) Tumors with high or low expression levels of p-mTOR and p-4E-BP1 pre- and post-NAC. (E and F) Tumors with high or low expression of mTOR post-NAC, in groups A and B. High levels of p-mTOR and p-4E-BP1 expression pre-NAC were found to correlate with a poor DFS, and a high expression of p-mTOR post-NACwas found to be significantly associated with a poor DFS. High expression of p-mTOR post-NAC was found to correlate with a poor DFS, regardless its expression pre-NAC. Group A, high expression of p-mTOR pre-NAC; group B, low expression of p-mTOR pre-NAC; p-mTOR, phosphorylated-mammalian target of rapamycin; p-4E-BP1, phosphorylated-eukaryotic translation initiation factor 4E-binding protein 1; NAC, neoadjuvant; DFS, disease-free survival.

significant correlations were found between the two factors and other clinicopathological features.

High levels of $p-m T O R$ and $p-4 E-B P 1$ pre-NAC correlate with poor DFS, and the high expression of p-mTOR post-NAC has a significant association with poor DFS. In order to assess the differential survival with respect to pre- and post-NAC expression, as well as the expression change following NAC for the two markers, Kaplan-Meier survival analyses were performed. ROC curve analyses were used to dichotomize the expression scores into high and low expression groups. The cut-off values were obtained from the highest combined sensitivity and specificity at the endpoint of DFS, and were as follows: p-mTOR, 8 and p-4E-BP1, 9 for pre-NAC; and p-mTOR, 7 and p-4E-BP1, 5 for post-NAC. A high expression of p-mTOR and p-4E-BP1 was found to significantly correlate with poor DFS pre-NAC (Fig. 4A and B, log-rank, $\mathrm{P}=0.013$ for $\mathrm{p}-\mathrm{mTOR}$ and $\mathrm{P}=0.025$ for $\mathrm{p}-4 \mathrm{E}-\mathrm{BP} 1)$. The expression of p-4E-BP1 post-NAC was not significantly correlated with DFS (Fig. 4D). By contrast, the high expression of
p-mTOR in post-NAC samples had a significant association with poor DFS compared with the pre-NAC samples (Fig. 4C, log-rank, $\mathrm{P}<0.001)$. It was also examined whether the expression changes of the factors correlate with DFS. Changes in expression of the two factors were dichotomized as up- or downregulated, but no significant correlation was found. In order to identify the predictive value of mTOR post-NAC, patients with high p-mTOR expression pre-NAC were defined as group A, while those with low p-mTOR expression pre-NAC were defined as group B. Next, the p-mTOR expression was compared between groups A and B post-NAC. High expression of $\mathrm{p}$-mTOR post-NAC was found to correlate with poor DFS, regardless of whether the patients were in group A or B ( $\mathrm{P}=0.043$ for group A and $\mathrm{P}=0.006$ for group B). Finally, multivariate Cox regression analysis was performed taking into account p-mTOR and p-4E-BP1 expression pre-NAC, p-mTOR expression post-NAC and other clinicopathological features, including tumor grade, receptor status, tumor stage pre-NAC and axillary metastasis. Post-NAC expression of p-mTOR was identified as the only significant factor, with its 
high expression associated with a hazard ratio of $3.073(95 \%$ confidence interval, 1.4-6.8; $\mathrm{P}=0.006$ ).

\section{Discussion}

Consistent with previous studies, the expression levels of p-mTOR and p-4E-BP1 in pre-NAC samples were found to significantly correlate with each other in this study. However, following chemotherapy, the expression levels of the two factors were found to decrease and no longer showed a correlation. This may indicate that the PI3K/Akt/mTOR/4E-BP1 pathway can be suppressed by chemotherapy in certain patients without treatment with mTOR inhibitors. This result supports the previous finding that the expression of eIF4E, as the downstream factor of p-4E-BP1, was reduced following NAC (34). This may also suggest that chemotherapy can suppress certain upstream signals or regulators of mTOR, such as Akt, PTEN and TSC1/TSC2, resulting in the inhibition of the mTOR/4E-BP1/eIF4E pathway. HER2-mediated activation of the PI3K/Akt/mTOR pathway has been implicated in the angiogenesis and metastasis of breast cancers (35), and is predictive of tumor progression (2).In an in vitro study, HER2-overexpressing cells with an activated Akt/mTOR/4E-BP1 pathway were more dependent on this pathway for growth and, therefore, were more sensitive to mTOR inhibition (2). A previous study has also shown that the expression of $\mathrm{p}-\mathrm{mTOR}$ and $\mathrm{p}-4 \mathrm{E}-\mathrm{BP} 1$ correlate with HER2 expression, which is consistent with the finding in this study. The decrease of p-mTOR was also found to be significant in HER2-positive patients compared with HER2-negative patients, indicating that the PI3K/Akt/mTOR pathway may be suppressed more effectively by chemotherapy in HER2-positive breast cancers. Based on these results, we hypothesize that HER2-positive patients with high p-mTOR expression following NAC may benefit more from the addition of mTOR inhibitors to chemotherapy. However, further study is required to investigate the specific association between the $\mathrm{PI} 3 \mathrm{~K} / \mathrm{Akt} / \mathrm{mTOR} / 4 \mathrm{E}-\mathrm{BP} 1$ pathway and chemotherapy sensitivity.

It has been demonstrated that patients with favorable responses to NAC exhibit improved DFS (36). Previous clinical trials have also shown that patients who achieve pCR exhibit an improved DFS and OS (16-19). Although a number of different prognostic indicators are being developed, few molecular markers are widely used to predict the NAC responses. In this study, the change of p-mTOR expression was found to correlate with the change of tumor size. Patients with lower levels of p-mTOR expression following NAC are likely to have smaller tumor sizes. Although this finding is not useful to predict the sensitivity of chemotherapy prior to NAC, it can be used as a marker of the effect during the course of NAC and as a reference to decide the chemotherapy regimen following surgery. It was also noted that the PI3K/Akt/mTOR pathway correlates with the resistance to chemotherapy based on the analysis of the correlation between the change of p-mTOR expression and the change of tumor size.

This study not only confirmed previous findings that high levels of p-mTOR and p-4E-BP1 pre-NAC are significantly associated with poor DFS, but also found that the high expression of $\mathrm{p}-\mathrm{mTOR}$ in post-NAC samples exhibits a significant association with poor DFS. No significant correlation was found between the changes of the two factors, or between the post-NAC expression of 4E-BP1 and DFS. In an effort to investigate the reason why the change of p-mTOR was not found to correlate with DFS, it was demonstrated that the p-mTOR expression significantly correlated with DFS, regardless of whether its expression was high or low pre-NAC. This result indicated that patients whose tumors contain high levels of $\mathrm{p}$-mTOR following NAC may be more resistant to chemotherapy. These patients, particularly HER2-positive patients, may be more appropriate candidates for adding mTOR inhibitors to the chemotherapy. As a downstream factor of $\mathrm{p}-4 \mathrm{E}-\mathrm{BP} 1$, eIF4E has been demonstrated to correlate with DFS, regardless of its expression in neoadjuvant (34) or adjuvant chemotherapy (10). However, the p-4E-BP1 expression post-NAC or the change of p-4E-BP1 was not found to correlate with DFS. Although the exact mechanism is not clear, this may be due to the different chemotherapy regimens.

The expression levels of p-mTOR and p-4E-BP1 were significantly decreased following treatment with NAC, particularly in HER2-positive samples. However, little is known with regard to the mechanisms that drive the expression changes of the two factors. The p-mTOR expression post-NAC may be a more reliable predictor to DFS in NAC patients, and may be used as a reference to select patients that are suitable for adding mTOR inhibitors to the chemotherapy. It is known that mutations of PIK3CA, Akt and PTEN, or the loss of PTEN may influence the expression of p-mTOR following NAC. Therefore, these mutations may cause different sensitivities to chemotherapy. Further study is required to clarify the exact mechanisms and to evaluate the predictive value of the $\mathrm{PI} 3 \mathrm{~K} / \mathrm{Akt} / \mathrm{mTOR} / 4 \mathrm{E}-\mathrm{BP} 1$ pathway in NAC.

\section{Acknowledgements}

This study was supported by grants from the National Natural Science Foundation of China (nos. 81172199 and 81272920) and the Hi-Tech Research Development Program of China (863 Program; no. 2006AA02Z493).

\section{References}

1. Ma XM and Blenis J: Molecular mechanisms of mTOR-mediated translational control. Nature Rev Mol Cell Biol 10: 307-318, 2009.

2. Zhou X, Tan M, Stone Hawthorne V, et al: Activation of the Akt/mammalian target of rapamycin/4E-BP1 pathway by ErbB2 overexpression predicts tumor progression in breast cancers. Clinical Cancer Res 10: 6779-6788, 2004.

3. Meric-Bernstam F and Gonzalez-Angulo AM: Targeting the mTOR signaling network for cancer therapy. J Clin Oncol 27: 2278-2287, 2009

4. Faivre S, Kroemer G and Raymond E: Current development of mTOR inhibitors as anticancer agents. Nat Rev Drug Discov 5: 671-688, 2006.

5. Margariti N, Fox SB, Bottini A and Generali D: 'Overcoming breast cancer drug resistance with mTOR inhibitors'. Could it be a myth or a real possibility in the short-term future? Breast Cancer Res Treat 128: 599-606, 2011.

6. Gingras AC, Raught B and Sonenberg N: eIF4 initiation factors: effectors of mRNA recruitment to ribosomes and regulators of translation. Annu Rev Biochem 68: 913-963, 1999.

7. Hara K, Yonezawa K, Kozlowski MT, et al: Regulation of eIF-4EBP1 phosphorylation by mTOR. J Biol Chem 272: 26457-26463, 1997. 
8. von Manteuffel SR, Dennis PB, Pullen N, et al: The insulin-induced signalling pathway leading to S6 and initiation factor $4 \mathrm{E}$ binding protein 1 phosphorylation bifurcates at a rapamycin-sensitive point immediately upstream of p70s6k. Mol Cell Biol 17: 5426-5436, 1997.

9. Brunn GJ, Hudson CC, Sekulić A, et al: Phosphorylation of the translational repressor PHAS-I by the mammalian target of rapamycin. Science 277: 99-101, 1997.

10. Heikkinen T, Korpela T, Fagerholm R, et al: Eukaryotic translation initiation factor 4E (eIF4E) expression is associated with breast cancer tumor phenotype and predicts survival after anthracycline chemotherapy treatment. Breast Cancer Ress Treat 141: 79-88, 2013.

11. Coleman LJ, Peter MB, Teall TJ, et al: Combined analysis of eIF4E and 4E-binding protein expression predicts breast cancer survival and estimates eIF4E activity. Br J Cancer 100: 1393-1399, 2009.

12. Jefferies HB, Fumagalli S, Dennis PB, et al: Rapamycin suppresses 5'TOP mRNA translation through inhibition of p70s6k. EMBO J 16: 3693-3704, 1997.

13. Kim EK, Kim HA, Koh JS, et al: Phosphorylated S6K1 is a possible marker for endocrine therapy resistance in hormone receptor-positive breast cancer. Breast Cancer Res Treat 126 93-99, 2011.

14. Fuksa L, Micuda S, Grim J, Ryska A and Hornychova $\mathrm{H}$ : Predictive biomarkers in breast cancer: their value in neoadjuvant chemotherapy. Cancer Invest 30: 663-678, 2012.

15. Mieog JS, van der Hage JA and van de Velde CJ: Neoadjuvant chemotherapy for operable breast cancer. Br J Surg 94: 1189-1200, 2007.

16. Kuerer HM, Newman LA, Smith TL, et al: Clinical course of breast cancer patients with complete pathologic primary tumor and axillary lymph node response to doxorubicin-based neoadjuvant chemotherapy. J Clin Oncol 17: 460-469, 1999.

17. Ogston KN, Miller ID, Payne S, et al: A new histological grading system to assess response of breast cancers to primary chemotherapy: prognostic significance and survival. Breast 12: 320-327, 2003 .

18. Thomas E, Holmes FA, Smith TL, et al: The use of alternate, non-cross-resistant adjuvant chemotherapy on the basis of pathologic response to a neoadjuvant doxorubicin-based regimen in women with operable breast cancer: long-term results from a prospective randomized trial. J Clin Oncol 22: 2294-2302, 2004

19. Heys SD, Hutcheon AW, Sarkar TK, et al: Neoadjuvant docetaxel in breast cancer: 3-year survival results from the Aberdeen trial. Clin Breast Cancer 3 (Suppl 2): S69-S74, 2002.

20. Guertin DA and Sabatini DM: Defining the role of mTOR in cancer. Cancer Cell 12: 9-22, 2007.

21. Dillon RL, White DE and Muller WJ: The phosphatidyl inositol 3-kinase signaling network: implications for human breast cancer. Oncogene 26: 1338-1345, 2007.

22. Cancer Genome Atlas Network: Comprehensive molecular portraits of human breast tumours. Nature 490: 61-70, 2012.
23. Banerji S, Cibulskis K, Rangel-Escareno C, et al: Sequence analysis of mutations and translocations across breast cancer subtypes. Nature 486: 405-409, 2012.

24. Saal LH, Holm K, Maurer M, et al: PIK3CA mutations correlate with hormone receptors, node metastasis, and ERBB2, and are mutually exclusive with PTEN loss in human breast carcinoma. Cancer Res 65: 2554-2559, 2005.

25. Stemke-Hale K, Gonzalez-Angulo AM, Lluch A, et al: An integrative genomic and proteomic analysis of PIK3CA, PTEN, and AKT mutations in breast cancer. Cancer Res 68: 6084-6091, 2008.

26. Mondesire WH, Jian W, Zhang H, et al: Targeting mammalian target of rapamycin synergistically enhances chemotherapy-induced cytotoxicity in breast cancer cells. Clinical Cancer Res 10: 7031-7042, 2004

27. Jerusalem G, Fasolo A, Dieras V, et al: Phase I trial of oral mTOR inhibitor everolimus in combination with trastuzumab and vinorelbine in pre-treated patients with HER2-overexpressing metastatic breast cancer. Breast Cancer Res Treat 125: 447-455, 2011.

28. Andre F, Campone M, O'Regan R, et al: Phase I study of everolimus plus weekly paclitaxel and trastuzumab in patients with metastatic breast cancer pretreated with trastuzumab. J Clin Oncol 28: 5110-5115, 2010.

29. Huober J, Hanusch C, Fasching PA, et al: Neoadjuvant chemotherapy of paclitaxel with or without Rad001: Results of the non-responder part of the GEPARUINTO study. Presented at 33rd Annual San Antonio Breast Cancer Symposium. San Antonio, TX, 2011

30. Yardley DA: Combining mTOR inhibitors with chemotherapy and other targeted therapies in advanced breast cancer: Rationale, clinical experience, and future directions. Breast Cancer (Auckl) 7: 7-22, 2013.

31. Yang F and Li J: WHO classification of tumors of the breast. Zhonghua Wai Ke Za Zhi 52: 1-3, 2014 (In Chinese).

32. Chen J and Fang Y: A novel pathway regulating the mammalian target of rapamycin (mTOR) signaling. Biochemical Pharmacol 64: 1071-1077, 2002.

33. Mita MM, Mita A and Rowinsky EK: The molecular target of rapamycin (mTOR) as a therapeutic target against cancer. Cancer Biol Ther 2 (4 Suppl 1): S169-S177, 2003.

34. Hiller DJ, Chu Q, Meschonat C, et al: Predictive value of eIF4E reduction after neoadjuvant therapy in breast cancer. J Surg Res 156: 265-269, 2009

35. Klos KS, Wyszomierski SL, Sun M, et al: ErbB2 increases vascular endothelial growth factor protein synthesis via activation of mammalian target of rapamycin/p70S6K leading to increased angiogenesis and spontaneous metastasis of human breast cancer cells. Cancer Res 66: 2028-2037, 2006.

36. Hanrahan EO, Hennessy BT and Valero V: Neoadjuvant systemic therapy for breast cancer: an overview and review of recent clinical trials. Expert Opin Pharmacother 6: 1477-1491, 2005. 